\title{
TOBACCO USE AND THE CARDIOVASCULAR DISEASE EPIDEMIC IN DEVELOPING COUNTRIES: PREVENTION OPPORTUNITY.
}

Matheka C. Wambua ${ }^{1,2}$ and Imran Jamal ${ }^{1,2}$

${ }^{1}$ Department of Medical Physiology, University of Nairobi (UoN) ${ }^{2}$ School of Medicine (UoN), Kenya.

DMSJ 2012;19: 17-21 http://dx.doi.org/10.4314/dmsj.v19i1.4

\begin{abstract}
Introduction: Cardiovascular diseases remain the leading cause of death in the world, far outstripping deaths due to malaria, Human Immunodeficiency Virus/Acquired Immune Deficiency Syndrome (HIV/AIDS) and tuberculosis. Approximately $80 \%$ of the 35 million deaths annually are attributable to chronic diseases and a similar proportion of the approximately 16.7 million deaths due to CVD occur in low and middle income countries. Tobacco use is one of the major avoidable causes of cardiovascular disease.

Context and objectives: Smoking is the leading cause of preventable death and is associated with an increased risk of various diseases including cardiovascular disease. The aim of this paper is to review the literature on the role of smoking on cardiovascular disease and effects of antismoking legislation on admission due to acute myocardial infarction.

Research method/Methodology: PubMed and manual search strategies using the search words 'tobacco and cardiovascular disease' were used to identify articles published on the contribution of smoking to cardiovascular events and the effects of legislation. 798 articles were found of which 28 were found useful and 39 were found to be highly relevant for the review.

Results: Smoking has been significantly related to acute myocardial infarction ( $<<0 \cdot 0001)$ with an odds ratio of $2.87(2 \cdot 36-3 \cdot 48)$ for current $v s$. never, PAR $35.7 \%$ for current and former $v s$. never). Smoking bans have been associated with decreases in instances of acute myocardial infarction ranging from a $40 \%$ decrease found in Helena, Montana, USA to an $8 \%$ reduction seen in the State of New York.

Conclusion: Cardiovascular diseases are a major public health challenge. Smoking plays a major role in cardiovascular pathophysiology. Therefore efforts are required to address the problem especially at preventive level. Public smoking bans are related to fewer admissions for both cardiovascular and respiratory conditions. Legislative efforts to reduce exposure to smoking are needed especially in the developing countries, where prevalence of smoking is rising.
\end{abstract}

Corresponding author: Matheka C. Wambua, Tel: +254726933731, E-mail address: mcw102005@yahoo.co.uk

\section{INTRODUCTION}

CardioVascular Disease (CVD) remains the leading cause of death in the world, outstripping deaths due to malaria, Human Immunodeficiency Virus/ Acquired Immune Deficiency Syndrome and tuberculosis combined. Approximately $80 \%$ of the 35 million deaths annually attributable to chronic diseases are attributable to CVD. A similar proportion of the 16.7 million deaths due to CVD occur in low and middle income countries (LMIC's) (1). The increasing 
incidence of CVD in LMIC's adds to the continuing burden of infectious, nutritional, and perinatal diseases, the "African double-burden of disease", (2).

Although age-adjusted cardiovascular death rates have declined in several developed countries in past decades, CVD incidence has risen in LMIC's (2). Effective prevention needs strategic planning and policy formulation based on knowledge of the burden and the importance of risk factors.

Cigarette smoking is the leading cause of preventable morbidity and premature death in developed countries (3).Compared with non-smokers, smokers are 15 times more likely to develop lung cancer, 11 times more likely to develop chronic lung disease and twice as likely to have acute myocardial infarctions (AMI's) (4).

Smoking causes premature death and disease in people who do not smoke but are passively exposed to environmental tobacco smoke, the so-called second-hand smoke (SHS). Studies indicate that exposure to passive smoking is associated with a 30\% increased risk of AMI (5). In a meta-analysis that included 29 studies, SHS increased the risk for AMI by $31 \%$ compared with the doubling of risk associated with direct smoking (6). Recent evidence, based on more accurate biological markers of exposure, suggests that the risk of passive smoking could be increased by as much as $60 \%$, similar to that observed in light active smokers. Second-hand smoke is the third leading cause of preventable poor health and premature death in the developed world (7).It's a recognized risk factor for ischemic heart disease (8). It may act as a cardiovascular trigger (9), as supported by several studies linking exposure to passive smoking to various acute cardiovascular effects, including platelet activation $(10,11)$, impaired endothelial function $(12,13,14)$, arterial stiffness (15), reduced heart rate variability (16), and increased levels of venous carboxyhemoglobin (17).

Although smoking bans have been shown to reduce CVDs, sub-Saharan Africa lacks strong legislative policies to control the use of such products. This is coupled with high illiteracy levels and aggressive advertising by the tobacco multinational companies. The strength of these multinationals in relation to the country economies further complicates the scenario. To the taxcollector, income from tobacco products makes a major contribution to the national revenue base. This is in addition to the thousands of jobs that the sector creates. The aim of this review was to determine the contribution of smoking to the CVD burden and the effects of smoking bans on the admission to hospital for acute myocardial infarction and other cardiovascular events.

\section{RESEARCH METHODOLOGY}

PubMed database plus bibliographies of relevant studies and reviews were searched for peer reviewed articles published using the words 'tobacco and cardiovascular disease' to identify articles published on the contribution of smoking to cardiovascular events and the effects of antismoking legislation. Seven hundred and ninety eight (798) articles were found of which twenty eight (28) were found useful and thirty nine (39) were found to be highly relevant for the review. Relevant bibliographies were also included.

\section{RESULTS}

The INTERHEART study identified the modifiable risk factors for CVD in both sexes and at all ages worldwide: smoking, raised ApoB/ApoA1 ratio, history of hypertension and diabetes, 
abdominal obesity, psychosocial factors, lower daily consumption of fruits and vegetables, regular alcohol consumption, and physical inactivity.

\section{Prevalence of Smoking Globally}

Epidemiologic studies strongly support the assertion that cigarette smoking in both men and women increases the incidence of AMI and fatal coronary artery disease (19). SHS increases the risk of AMI from $25 \%$ to $31 \%$ (6). In countries where smoking prevalence is high, for example, Britain (50\%) (20), Europe (62\%) (21), and Greece (156\%) (22), compared to $22 \%$ in the USA (23), AMI in non-smokers is particularly increased.

Sub-Saharan Africa (SSA) appears to differ from other regions of the world in having reached only the early stages of the cigarette smoking epidemic. Estimates suggest that deaths from smoking-attributed causes reach only $5-7 \%$ for men and 1-2\% for women. Comprehensive data on the prevalence of smoking in SSA is largely missing. However, data from demographic health surveys shows different smoking prevalence rates: Nigeria (8.0\%), Ethiopia (8.3\%), Ghana (8.8\%), Mozambique (14.1\%), Lesotho (15.6\%), Zambia (15.6\%), Namibia (17.5\%), Zimbabwe (22.1\%), Rwanda (14.2\%), Uganda (18.1\% and 18.7\%), and Tanzania (21\%) (24). Kenya $(22.9 \%)$ generally has a higher prevalence of cigarette smoking. Malawi has moderate to high prevalence but experienced a small decline from $18.7 \%$ to $16.6 \%$ from 2000 to 2004 . Madagascar stands out as having the highest cigarette smoking prevalence of all the nations $(27.3 \%)$. The range of the prevalence of smoking (8.0-27.3\%) demonstrates considerable diversity (25). The INTERHEART study reported a current smoking prevalence of $52.9 \%$ and $27.2 \%$ in young males and females respectively, and $38.1 \%$ and $8.1 \%$ in older males and females respectively (21).

\section{Smoking and the Cardiovascular System (Pathophysiology and Risk)}

Passive smoking is a recognized risk factor for cardiovascular heart disease. It may act as a cardiovascular trigger (9) as supported by several studies linking exposure to passive smoking to various acute cardiovascular effects, including platelet activation (11), impaired endothelial function (14), arterial stiffness (15), reduced heart rate variability (16), and increased levels of venous carboxyhemoglobin. These acute effects are probably transient and disappear in a few hours after cessation of the exposure $(6,13,14,16)$.

Cigarette smoking predisposes the individual to several different clinical atherosclerotic syndromes including stable angina, acute coronary syndromes, sudden death and stroke. Aortic and peripheral atherosclerosis is also increased, leading to intermittent claudication and abdominal aortic aneurysms (26). The mechanisms involved include;

a) Inflammation: The inflammatory response is an essential component in the initiation and evolution of atherosclerosis. Several studies have indicated that cigarette smoking causes about a $20 \%$ to $25 \%$ increase in the peripheral blood leukocyte count. In vivo, cigarette smoking is associated with an increased level of multiple inflammatory markers such as C-reactive protein, interleukin-6, and tumour necrosis factor alpha in both sexes $(27,28,29,30)$. 
b) Modification of the lipid profile: Cigarette smoking could promote atherosclerosis, in part, by its effects on the lipid profile. Smokers have significantly higher serum cholesterol, triglyceride, and low-density lipoprotein (LDL) levels, but high-density lipoprotein is lower in smokers than in non-smokers (31). The mechanisms responsible are not clearly elucidated, and the role of dietary differences between smokers and non-smokers is unknown. The triglyceride/high-density lipoprotein abnormalities have lately been suggested to be related to insulin resistance (32).

Other mechanisms that have been proposed to be related to cardiovascular pathophysiology include vasomotor dysfunction, increased prothrombotic and decreased fibrinolytic factors, leukocyte activation, and smooth muscle proliferation. These mechanisms may be triggered by decreased nitric oxide bioavailability, increased oxidative stress and production of proinflammatory cytokines (33).

In the INTERHEART study, current smoking was associated with a three-fold increase in odds of a non-fatal AMI, compared to people that never smoked before (odds ratio [OR] 2.95, 95\% CI 2.77-3.14, $\mathrm{p}<0.0001)$. The risks were higher depending on the number of cigarettes smoked, with people who smoked one to nine cigarettes per day having an OR of 1.63 (95\% CI 1.45$1.82, \mathrm{p}<0.0001), 10-19$ per day an OR of $2.59(2.35-2.85, \mathrm{p}<0.0001)$, while those who smoked 20 or more per day had an OR of 4.59 (4.21-5.00, $\mathrm{p}<0.0001)$. Similar trends were noted in both men and women. Women who smoked 1-19 cigarettes per day had an OR of 2.11 (1.77-2.52, $\mathrm{p}<0.0001)$ while those who smoked 20 or more per day had an OR of 5.11 (3.98-6.56, $\mathrm{p}<0.0001)$ for AMI. For men, the corresponding OR's were $2.06(1.90-2.24, \mathrm{p}<0.0001)$ and 4.48 $(4.13-4.87, \mathrm{p}<0.0001)$ respectively. The effect of current smoking was much larger in younger (OR 3.53, 95\% CI 3.23-3.86) than in older individuals $(2.55,22.35-2.76 ; \mathrm{p}<0.0001$ for interaction); especially in heavy smokers ( $\geq 20$ cigarettes per day) in whom OR's were 5.60 (95\% CI 5.1-6.20) for younger and 3.60 (3.25-3.98, $<<0.0001$ for interaction) for older smokers (21). Lorenzo et al., (34), reported that in a population with an average of 5 hours per week of overall exposure to passive smoking, a 50\% reduction in this exposure after the introduction of antismoking regulation, translates into $8.6 \%$ decrease in AMI in passive and active smokers.

\section{Smoking bans and effect on admission secondary to AMI}

Smoking bans in different parts of the world have been associated with fewer hospital admissions due to AMI. In Helena Montana; a smoking ban decreased the hospital admission by $40 \%$ from 170 to 102 cases $/ 100,000$. In surrounding areas where bans had not been effected, incidence increased by $46 \%$ from 118 to 172 cases/100,000 person-years. In Pueblo (Colorado), AMI decreased by $27 \%$ (257 to 187 cases/100,000 person-years) during a ban period. A state public smoking ban in New York coupled to increased taxation on tobacco before July 2003 led to an $8 \%$ decrease of AMI-related admissions from 483 to 445 cases/100,000 person-years (4, 35, 36). In Indiana smoking bans in all restaurants, retail outlets and work places in 2003 decreased the AMI incidence from 14 to 7 cases/100,000 person-years. The observations were mainly from non-smokers, elaborating the contribution of passive smoking to cardiovascular pathophysiology. In Bowling Green (Ohio), smoking bans in work places and public areas except bars reduced AMI incidence from 277 to 223 cases/100,000 person-years (35). 
Saskatoon (Canada) implemented a smoking ban in all enclosed public areas and outdoor seating places in July 2004. The incidence of AMI decreased by 14\% from 176 to 152 cases/100,000 person-years (35). In Piedmont (Italy), smoking in cafes, restaurants, bars and discos was banned in 2005. Subsequently nicotine vapour use in public areas decreased by $90-95 \%$, cigarette sales declined by $8.9 \%$ while cigarette consumption decreased by $7.6 \%$. The study suggested a greater effect of the ban on young people and a lower risk of AMI from smoking among older people (35). Incidence of AMI decreased significantly among 35-64 year old men (IRR=0.89) and in 6574 year old men (IRR 1.02). Among women (30\% of the cases of AMI), the decrease was confined to the young people (IRR 0.90 in 35-64 year old women) following a smoking ban in Rome, Italy. Since March 2006, smoking has been prohibited in all enclosed public areas in Scotland. After the ban, a $17 \%$ decrease in cases of acute coronary syndrome was reported. Admission decreased by $14 \%$ in smokers, $19 \%$ in former smokers and $21 \%$ in people that never smoked before. It is estimated that $67 \%$ of the admissions prevented involved non smokers with a decrease from $43 \%$ to $22 \%$ in reported decrease in second hand smoking. In a meta-analysis comparing AMI before and after smoking bans, an overall IRR of 0.83 indicated that smoking bans on an average reduced AMI incidence by $17 \%$.

In England, emergency admissions for AMI after the implementation of smoke-free legislation dropped by $2.4 \%$. This equates to 1200 fewer emergency admissions for myocardial infarction (1600 including readmissions) in the first year after legislation. The reduction in admissions was significant in men and women aged 60 and over, and men (3.5\%) but not women (2.5\%) aged under 60 (37).

There is, however, paucity of data on the effect of smoking bans on AMI admissions in developing countries.

\section{DISCUSSION}

Tobacco use is associated with an increased risk for AMI. The risk is greater for the young than for the old. The magnitude of risk is closely and linearly, related to the number of cigarettes smoked, with even low levels of smoking (e.g., five cigarettes per day) being associated with an appreciable risk of AMI. Former smokers have a higher risk of AMI than non-smokers, but this risk decreases with time after stopping smoking. A large part of the excess risk of AMI associated with smoking dissipates within five years, and among light smokers there was no excess risk after 3-5 years of quitting. By contrast, moderate and heavy smokers still have an increased risk even 20 years after quitting. Exposure to SHS increases the risk of AMI, in nonsmokers and former smokers (21). The INTERHEART study provides useful data on the association between tobacco use and risk of AMI from all regions of the world. The similarity of the increased risks in all regions lends strong support to the need for a global approach to tobacco control.

The focus of anti-tobacco programmes must not only prevent young adults from starting smoking, but also promote quitting in current smokers. The latter are at high risk of developing a major vascular event (or tobacco-related cancer) in the future. The adverse health effects of exposure can take many years to appear, whereas the benefits of withdrawal appear much more rapidly. Therefore, a strategy to facilitate quitting in current middle-aged smokers is likely to have a substantial health benefit within a relatively short time (18). 
While developed countries have well developed tobacco control policies, LMIC's lack the same. The tobacco industry has expended great efforts to create uncertainty about the harm caused by its product. Consequently, public and political support for implementation of effective tobacco control policies is low in LMIC's. Nevertheless, the INTERHEART data provide support for intensifying tobacco control policies worldwide (18).

Studies on the effects of smoking on the cardiovascular system have potential limitations. For example, the INTERHEART study involved participants who had survived AMI and by using a case-control design could not elucidate the relationship between tobacco use and risk of AMI in individuals who died in the early phase of the AMI or because of serious complications or comorbidity. Patients with AMI might over-report exposure to tobacco smoke. However, other reports suggest that compared with biochemical monitoring with nicotine levels, self reporting in AMI survivors tends to underestimate the prevalence of smoking (38). In any case, self reporting of tobacco use is thought to be reliable and the results are similar to those of other studies with different designs, such as cohort studies. However the relative risk for AMI of about 3.00 for AMI survivors in the INTERHEART study is consistent with the relative total mortality risk of 2.19 for current smokers in the British Doctors study (39).

\section{CONCLUSION}

CVD's are a major health challenge. Smoking plays a major role in cardiovascular pathophysiology and efforts are required to address the problem especially at the preventive level. Different measures including public education and legislations have proved effective in different parts of the developed countries by reducing emergency admission in hospital. Legislated bans on smoking are associated with reduced rates of admission to hospital, reinforcing the value of such bans to public health. Consistent evidence also reports that exposure to second-hand smoke is detrimental to health. This review adds to a growing body of evidence that smoke-free legislation leads to reductions in the incidence of myocardial infarction. Developing countries should use this evidence-based body of knowledge to make policies that will reduce the increased trend of smoking among the population.

\section{DISCLOSURE}

This review article was presented during the $4^{\text {th }}$ International TAMSA conference in Dar-esSalaam in 2010. The article has not been submitted to any other journal for publication. The authors declare no conflict of interest.

\section{References}

1. Fuster V. and Voûte J. MDG: chronic diseases are not on the agenda. Lancet 2005; 366:1512-1514.

2. Salim Y., Srinath R., Stephanie Ô., Sonia A. Global Burden of Cardiovascular Diseases Part I: General Considerations, the Epidemiologic Transition, Risk Factors, and Impact of Urbanization Circulation 2001; 104:2746-2753. 
3. Demosthenes B.P., Christos P., Christina C., John S., Constadina M., Pavlos T., Christodoulos S. Effect of Exposure to Second-hand Smoke on Markers of Inflammation: the ATTICA Study. Am J Med. 2004; 116:145-150.

4. David G.M. and John S.N. Cardiovascular Effect of Bans on Smoking in Public Places. Am J Cardiol 2008; 102:1421-1424.

5. Law M.R. and Wald N.J. Environmental tobacco smoke and ischemic heart disease. Prog. Cardiovasc. Dis 2003; 46: 31-38.

6. Barnoya J. and Glantz S.A. Cardiovascular effects of secondhand smoke: nearly as large as smoking. Circulation 2005; 111: 2684-2698.

7. Glantz S. and Parmley W.W. Passive smoking and heart disease: epidemiology, physiology, and biochemistry. Circulation 1991; 83:1-12.

8. Office of Environmental Health Hazard Assessment, California Environmental Protection Agency, 1997. Health effects assessment for environmental tobacco smoke.

9. Raupach T., Schafer K., Konstantinides S., Andreas S. Second-hand smoke as an acute threat for the cardiovascular system: a change in paradigm. Eur Heart J 2006; 27: 386-392.

10. Burghuber O.C., Punzengruber C., Sinzinger H., Haber P., Silberbauer K. Platelet sensitivity to prostacyclin in smokers and non-smokers. Chest 1986; 90: 34-38.

11. Schmid P., Karanikas G., Kritz H., et al. Passive smoking and platelet thromboxane. Thromb. Res. 1996; 81: 451-460.

12. Celermajer D.S., Adams M.R., Clarkson P., et al. Passive smoking and impaired endothelium-dependent arterial dilatation in healthy young adults. N Engl J Med 1996; 334: $150-154$.

13. Lekakis J., Papamichael C., Vemmos C., Stamatelopoulos K., Voutsas, A., Stamatelopoulos S., Effects of acute cigarette smoking on endothelium-dependent arterial dilatation in normal subjects. Am. J. Cardiol 1998; 81: 1225-1228.

14. Argacha J.F., Adamopoulos D., Gujic M. et al., Acute effects of passive smoking on peripheral vascular function. Hypertension 2008; 51: 1506-1511.

15. Stefanadis C., Vlachopoulos C., Tsiamis, E., et al. Unfavorable effects of passive smoking on aortic function in men. Ann. Intern. Med 1998; 128: 426-434.

16. Pope C.A., Eatough, D.J., Gold D.R., et al., Acute exposure to environmental tobacco smoke and heart rate variability. Environ. Health Perspect. 2001; 109: 711-716.

17. Aronow W.S. Effect of passive smoking on angina pectoris. N Engl J Med 1978; 299: 21-24.

18. Salim Y., Steven H., Stephanie Ô., Tony D., Alvaro A., Fernando L., Matthew M., Andrzej B., Prem P., John V., Liu L. Effect of potentially modifiable risk factors associated with myocardial infarction in 52 countries (the INTERHEART study): case-control study. Lancet 2004; 364: 937-52

19. Price J.F., Mowbray P.I., Lee A.J., Rumley A., Lowe G.D., Fowkes F.G. Relationship between smoking and cardiovascular risk factors in the development of peripheral arterial disease and coronary artery disease: Edinburgh Artery study. Eur Heart J 1999; 20:344-53.

20. Whincup P.H., Gilg J.A., Emberson J.R., et al. Passive smoking and risk of coronary heart disease and stroke: prospective study with cotinine measurement. BMJ 2004; 329:200 -5. 
21. Teo K.K., Ounpuu S., Hawken S., et al. Tobacco use and risk of myocardial infarction in 52 countries in the INTERHEART study: a case control study. Lancet 2006; 368:647-58.

22. Pitsavos C., Panagiotakos D.B., Chrysohoou C., et al. Association between exposure to environmental tobacco smoke and the development of acute coronary syndromes: the CARDIO2000 case-control study. Tob Control 2002; 11:220 -5.

23. U.S. Department of Health and Human Services. Introduction, summary, and conclusions. In: The Health Consequences of Involuntary Exposure to Tobacco Smoke. A Report of the Surgeon General. Atlanta, GA: U.S. Department of Health and Human Services, Centers for Disease Control and Prevention, Office of Smoking and Health, 2006:1-15.

24. Jagoe K., Edwards R., Mugusi F., Whiting D., Unwin N. Tobacco smoking in Tanzania, East Africa: population based smoking prevalence using expired alveolar carbon monoxide as a validation tool. Tobacco Control 2002; 11:210-214.

25. Fred P. Tobacco use in sub-Sahara Africa: Estimates from the demographic health surveys. Soc Sci Med. 2008; 66 : ( 8) 1772-1783.

26. Black H.R. Smoking and cardiovascular disease. In: Laragh JH, Brenner BM, editors. Hypertension: Pathophysiology, Diagnosis and Management. 2nd edition. New York, NY: Raven Press Ltd. 1995:2621-47.

27. Tracy R.P., Psaty B.M., Macy E., et al. Lifetime smoking exposure affects the association of C-reactive protein with cardiovascular disease risk factors and subclinical disease in healthy elderly subjects. .Arterioscler Thromb Vasc Biol 1997;17:2167-76.

28. Bermudez E.A., Rifai N., Buring J.E., Manson J.E., Ridker P.M. Relation between markers of systemic vascular inflammation and smoking in women. Am J Cardiol 2002; 89:1117-9.

29. Mendall M.A., Patel P., Asante M., et al. Relation of serum cytokine concentrations to cardiovascular risk factors and coronary heart disease. Heart 1997; 78:273-7.

30. Tappia P.S., Troughton K.L., Langley-Evans S.C., Grimble R.F. Cigarette smoking influences cytokine production and antioxidant defenses. Clin Sci (Lond) 1995; 88:485-9.

31. Craig W.Y., Palomaki G.E., Haddow J.E. Cigarette smoking and serum lipid and lipoprotein concentrations: an analysis of published data. BMJ 1989; 298:784-8.

32. Reaven G. and Tsao P.S. Insulin resistance and compensatory hyperinsulinemia: the key player between cigarette smoking and cardio-vascular disease. J Am Coll Cardiol 2003; 41:1044-7.

33. John A. A. and Rajat S.B. The Pathophysiology of Cigarette. Smoking and Cardiovascular Disease: An Update. J Am Coll Cardiol 2004; 43:1731-7.

34. Lorenzo R., Loredana V., Franco M., Francesco B. Cardiovascular benefits of smoking regulations: The effect of decreased exposure to passive smoking. Preventive Medicine 2009; 48: 167-172.

35. David G.M., John S.N., Jianghua H. Cardiovascular Effect of Bans on Smoking in Public Places. J Am Coll Cardiol 2009; 54:1249-55.

36. Carl B., Robert N. A., Christine N.W., William M.T., Raymond O.E., Becki B.B., Mori J. K. Reduction in the Incidence of Acute Myocardial Infarction Associated With a Citywide Smoking Ordinance. Circulation 2006;114;1490-1496. 
37. Michelle S., Roy M., Linda B., Anna G. Short term impact of smoke-free legislation in England: retrospective analysis of hospital admissions for myocardial infarction. BMJ 2010:340:c2161 doi:10.1136/bmj.c2161.

38. Woodward M. and Tunstall P.H. Biochemical evidence of persistent heavy smoking after a coronary diagnosis despite self-reported reduction: analysis from the Scottish Heart Health Study. Eur Heart J 1992; 13: 160-65.

39. Doll R., Peto R., Boreham J., Sutherland I. Mortality in relation to smoking: 50 years' observations on male British doctors. BMJ 2004; 328: 1519-28. 